\title{
On the characterization of a 1 meter long, ultra-precise KB-focusing mirror pair for European XFEL by means of slope measuring deflectometry
}

\author{
F. Siewert, ${ }^{1, a)}$ J. Buchheim, ${ }^{1}$ G. Gwalt, ${ }^{1}$ R. Bean, ${ }^{2}$ A.P. Mancuso, ${ }^{2}$ \\ ${ }^{1}$ Helmholtz Zentrum Berlin für Materialien und Energie, Department Optics and Beamlines, Albert-Einstein-Str. 15, \\ 12489 Berlin, Germany \\ ${ }^{2}$ European XFEL, Holzkoppel 4,22869 Schenefeld, Germany
}

\begin{abstract}
Recently the European XFEL has successfully produced its first X-ray photon pulse trains. This unique photon source will provide up to 27,000 photon pulses per second for experiments in different fields of science. In order to accomplish this, ultra-precise mirrors of dedicated shape are used to guide and focus these photons along beamlines of up to $930 \mathrm{~m}$ in length from the source in the undulator section to the desired focal point at an experimental station. We will report on a Kirkpatrick-Baez-mirror pair designed to focus hard-X-rays in the energy range from 3 to $16 \mathrm{keV}$ to a $100 \mathrm{~nm}$ scale at the SPB/SFX instrument of the European XFEL. Both mirrors are elliptical cylinder-like shaped. The figure error of these $1 \mathrm{~m}$ long mirrors was specified to be better than $2 \mathrm{~nm}$ pv in terms of the height domain this corresponds to a slope error of about $50 \mathrm{nrad}$ rms (at least a best effort finishing is requested). This is essential to provide optimal experimental conditions including preservation of brilliance and wave-front. Such large and precise optics represents a challenge for the required deterministic surface polishing technology, Elastic Emission Machining (EEM) in this case, as well as for the metrology mandatory to enable a precise characterization of the topography on the mirror aperture. Beside the slope errors the ellipse parameters are also of particular interest. The mirrors were under inspection by means of slope measuring deflectometry at the BESSY-NOM slope measuring profiler at the Helmholtz Zentrum Berlin (HZB). We will report on the measurement concept to characterize such mirrors as well as discussing the achieved results.
\end{abstract}

\section{Introduction}

Two elliptical cylinder like mirrors (often termed "plane ellipses") have been designed and manufactured to provide a $100 \mathrm{~nm}$ scale focusing in the horizontal and vertical directions respectively for the energy range between 3 to $16 \mathrm{keV}$ at the new SPB/SFX instrument [1,2] at European XFEL [3]. The mirror substrate dimensions are of $1000 \mathrm{~mm}$ in length and of $70 \times 70 \mathrm{~mm}^{2}$ in cross section. The substrate material is single crystal silicon having the 100 crystal orientation in the aperture plane. The aperture size is of $950 \times 25 \mathrm{~mm}^{2}$ located in the centered position on the substrate. The mirrors will be used in a Kirkpatrick-Baez (KB) configuration [4]. Such mirrors require an excellent finishing quality in the range of single nm pv figure errors and a $0.2 \mathrm{~nm}$ rms micro-roughness to allow a wave front preserving transport of photons $[5,6]$.

\footnotetext{
a) Author to whom correspondence should be addressed. Electronic mail: frank.siewert@helmholtz-berlin.de.
} 
Both mirrors were finished by deterministic surface finishing technology - Elastic Emission Machining (EEM) [7] in this case. For optimal reflectance the mirrors are coated with $\mathrm{B}_{4} \mathrm{C}[8,9]$ and Ruthenium on a second stripe [10] in two different 'stripes' to address the lower and higher photon energy ranges respectively. Importantly, these materials have been verified to withstand and not ablate under the expected incident fluence in operation at SPB/SFX [11]. To verify their usability, these two mirrors were under inspection regarding slope error and shape error as well as parameter of the ellipse. Due to their size and curvature along the meridional direction it is challenging to measure such mirror by means of a Fizeau interferometer. The method we have chosen is slope measuring deflectometry $[12,13]$. Slope measuring deflectometry, as provided by the well-known Long Trace Profiler [14] as well as a $2^{\text {nd }}$ generation slope measuring profiler [15] like the Nanometer Optic component Measuring machine (NOM) [16], is an established method in the synchrotron- and FEL-community to measure the quality of mirrors and gratings blanks in terms of slope error [17] as well as to gain the geometric substrate parameters like the radius of curvature or as in this case the parameter of the ellipse: entrance arm length, exit arm length and the grazing angle of the incidence X-ray beam. The knowledge of the ellipse parameter is essential to allow a precise installation of the mirrors at the vacuum vessel at the end-station and finally to provide the focal spot position as designed in space and size.

\section{The SPB/SFX instrument}

The Single Particles, Clusters and Biomolecules and Serial Femtosecond Crystallography (SPB/SFX) instrument of the European XFEL is designed predominantly to determine the structure of biological molecules either in the crystalline (through serial crystallography [18]) or non-crystalline (though small angle X-ray scattering [19] or single particle imaging [20]) states. The instrument $[2,21]$ is a forward scattering instrument with focal spots around the few micron and few hundred nanometer size to optimally illuminate samples of that scale. Mirror optics are chosen predominantly for their high transmission across the operating photon energy range [2], as well as for their resistance to ablation by the XFEL beam [18] and other optical properties. Samples are typically injected into the X-ray focus by means of a liquid jet for crystals or an aerosol jet for single particles [22]. A custom two-dimensional detector, called AGIPD [23] is then used to collect diffraction or scattering from the samples in question and that data can be interpreted to yield the bio-particles' structure. Particularly, an XFEL provides benefits for radiation damage sensitive samples, samples that are difficult to crystallize or only form small crystals, or to explore processes that evolve with time, such as mixing processes or photo-activated systems. The time resolved case is particularly 'data hungry' and the European XFEL with its many orders more pulses per unit time than other XFEL facilities [24] then becomes a most attractive facility to pursue such studies. 
The SPB/SFX instrument came online in 2017, accepted first users from September 2017 and had first results published in September $2018[25,26]$.

\section{SLOPE MEASURING DEFLECTOMETRY}

The BESSY-NOM is a $2^{\text {nd }}$ generation slope measuring profiler $[15,27]$ providing the characterization of reflective optical surfaces in the slope domain. Fig. 1 shows the principle of a slope measuring profiler with a fixed optics head and a scanning $45^{\circ}$ penta-prism set-up as first proposed by Debler and Zander [28] and is widely described in recent publications e.g. see the following references: [29-32]. Instead of a bulk penta prism we use a $45^{\circ}$ double mirror pair to avoid the inhomogeneity of the glass contributing as an error source. The detector used in the NOM is a modified autocollimator (AC) developed by Moeller Wedel Optical (MWO) $[33,34]$ fixed mounted on a stone pillar. The double mirror pair is mounted on an air bearing based carriage for guiding a laser test beam $(\lambda=650 \mathrm{~nm}$ generated by a LED) [35] along the optics under test, see also Fig. 1. Special care is required regarding a precise alignment of the double mirror set-up with respect to the autocollimator and carriage movement $[36,37]$. In addition the AC of the NOM was subject of dedicated calibration [27,38]. The laser beam is traced at regular intervals over the mirror along the line of inspection. In this case all measurements have been performed in the "on-the-fly" mode to save measurement time. To suppress drift-effects contributing to the measurement result all measurements were performed in a forward-backward mode, which allows for the compensation of linear drift contributions [39]. A circular diaphragm placed at a distance of $3 \mathrm{~mm}$ from the optics under test defines the size of the measuring beam. The size of the beam limited diaphragm is the major factor that defines the instrument's spatial resolution. Different investigations in the past have shown a measurement beam diameter of $2.5 \mathrm{~mm}$ to be the optimal size in the case of plane or slightly curved optics under inspection [27, 40]. Investigations on the modulation transfer function (MTF) of the autocollimator have shown a spatial resolution of 1.2-1.7 mm can be achieved with the set-up of the NOM (taking the zero-crossing of the system's MTF as the resolution criterion) [41]. 


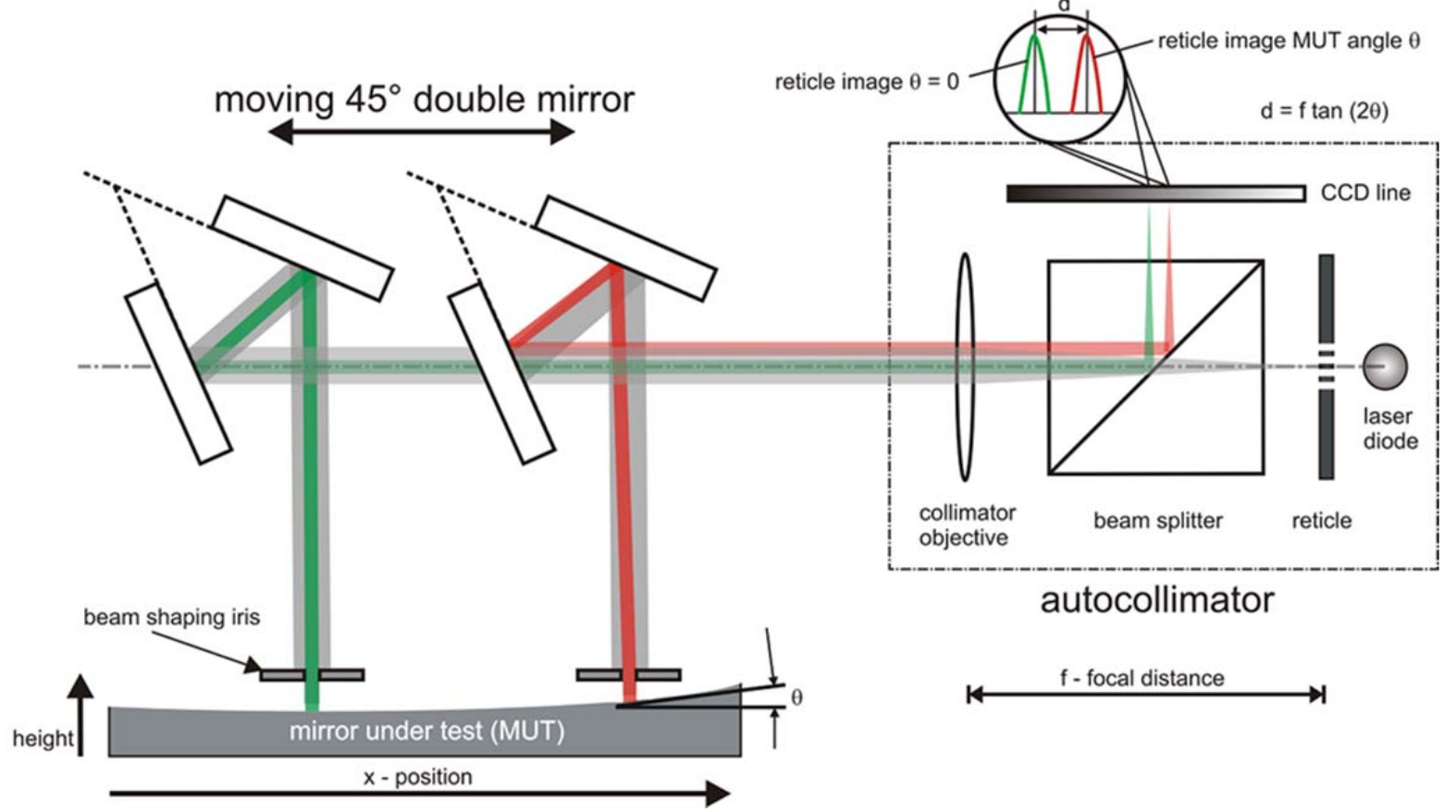

FIG.1. Principle set-up of a slope measuring profiler with scanning $45^{\circ}$-double-mirror as realized for the BESSY-NOM

With this arrangement (see Fig. 1) reflective surfaces of plane and curved shape, down to a local radius of curvature of $\mathrm{R}=4 \mathrm{~m}$, can be measured. Depending on the local topography of surface under test (SUT), the test beam will be reflected into the position sensitive detector (a CCD-line) of the NOM autocollimator head. Its position on the CCD-line of the sensor is directly related to the local surface slope. The reflection of the test beam along the optical axis of the instrument is determined by the angle between the mirror normal and the direction of the incident laser beam [42]. Then the local slope is given by:

$$
S(x)=\tan \theta=d y / d x
$$

The relative slope change is measured by scanning along the line of inspection. The autocollimator detects the change of the angle of reflection from one position $x$ on the mirror substrate to the next position $x+\Delta x$. A spatial integration of the slope data finally gives the topography height profile $h\left(x_{k}\right)$ : 


$$
h\left(x_{k}\right)=h\left(x_{0}\right)+\sum_{m=1}^{k} \frac{d x}{2}\left[S\left(x_{m}\right)+S\left(x_{m-1}\right)\right]
$$

\section{Measurements}

The two mirrors were aligned on a rotational stage with an $\mathrm{x}-\mathrm{y}$-tilting stage on top for angular fine alignment. A massive rectangular shaped plane rod of quartz glass was used to give support to the optics under test. The vertical focusing mirror was measured for two different conditions. First measurements were performed in full contact with a thin cellular foil between the mirror and the quartz glass rod to allow a full-contact all-over rest of the mirror. For the second run of measurements the mirror was placed on two gauge pieces of polished silicon - for this state see Fig. 2. These gauge pieces (made of polished Si-stripes) were placed at the design support position of the final mirror mechanics in the instrument. The rotational axis of the rotational stage was matching with the position of the mirror pole to allow a horizontal rotation of the optics by $180^{\circ}$ and measuring the mirrors in "side A to side B" and "side B to side A" orientation at the same x-coordinate range of the NOM.

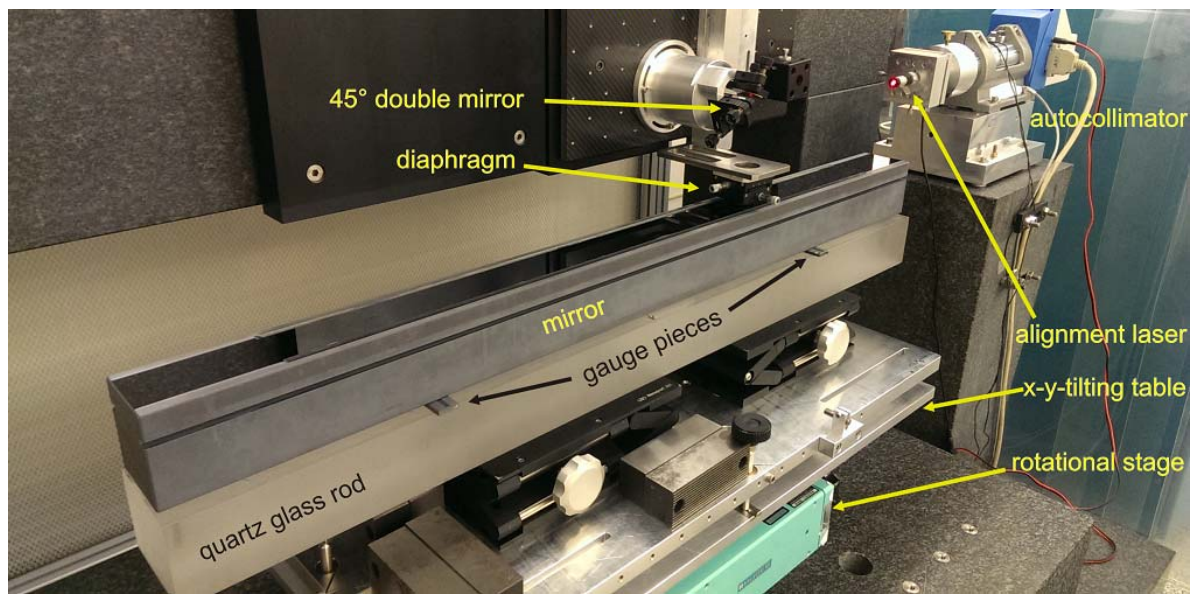

FIG.2. The vertical focusing mirror placed at gauge pieces during alignment at the BESSY-NOM.

The horizontal focusing mirror was measured in the full contact condition only as described previously for the vertical focusing mirror. We have chosen this option to avoid the complicated handling required (and hence avoid risk to these delicate optics) to perform measurements in the facing-to-the-side condition which is in principle possible at the BESSYNOM [43]. Earlier work [43] has shown the feasibility of this approach for characterizing optics to be used in facing-to-the- 
side condition. The difference between both cases was found to be in the range of sub-nm rms values. A first set of measurements was taken as line-scans along the central aperture axis in the meridional direction. With an increment of $\Delta \mathrm{x}=0.2 \mathrm{~mm}$. To suppress any systematic error contribution caused by the optical components of the autocollimator (lenses and split cube), as well as by variation of the measurement beam length and the inhomogeneity of the detector CCD-array, each mirror was measured at five different angular ranges of the autocollimator. Ten scans in "forward - backward" mode were taken for each angular range then the mirror was tilted and aligned to the next angular position. Averaging measurements taken in the "forward - backward" mode allows the suppression of the impact of linear drift effects on the measurement result [39]. Finally the data measured at five different angular ranges of the detector were averaged. This procedure was applied for the "upstream side to downstream side" measurements and after a rotation of $180^{\circ}$ for the "downstream side to upstream side" measurements. Fig. 3 shows the absolute slope data as obtained for the vertical focusing mirror with the mirror support at the gauge pieces.
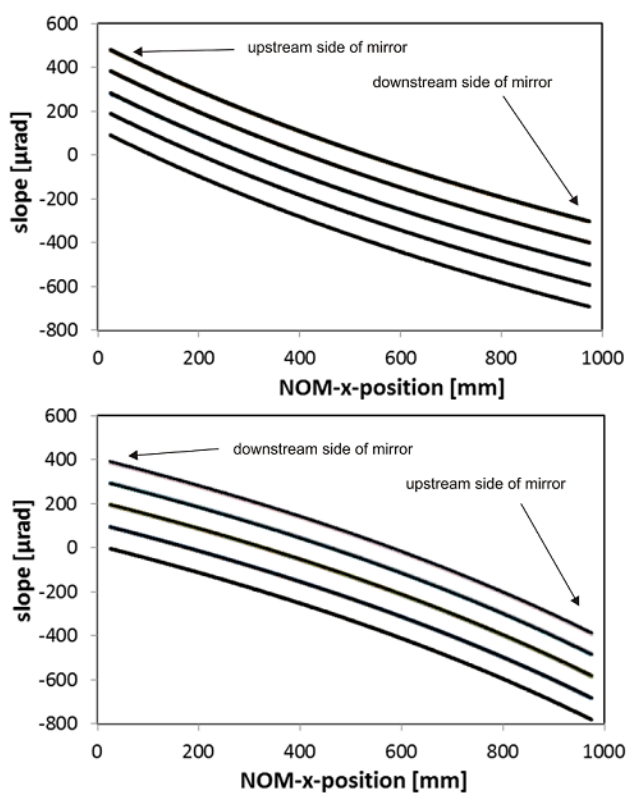

FIG.3. Slope data as measured at the NOM on the vertical focusing mirror (supported by gauge pieces) at five different angular ranges of the AC. Top: for the side "upstream side to downstream side" substrate orientation alignment. Bottom: for the side "downstream side to upstream side" alignment.

The final result is achieved by averaging the results gained for both orientations. The same procedure was applied to measure the horizontal focusing mirror. Such line-scans allow the identification of the slope error and residual figure error along this line of inspection. In addition one gets the parameter of the best-fit ellipse: entrance-arm- and exit-arm-length as 
well as the grazing angle. For this we have applied a best slope ellipse fit to the measured slope data as proposed by Sutter and co-worker [44], see equation 3.

$$
\begin{array}{r}
\sigma(x)=\frac{\left(r+r^{\prime}\right) \sin \theta}{\left(r+r^{\prime}\right)^{2}-\left(r-r^{\prime}\right)^{2} \sin ^{2} \theta}\left(2 r r^{\prime}-\left[\left(r-r^{\prime}\right) \cos \theta\right] x\right. \\
\left.\quad-2\left(r r^{\prime}\right)^{1 / 2}\left\{r r^{`}-\left[\left(r-r^{\prime}\right) \cos \theta\right] x-x^{2}\right\}^{1 / 2}\right)
\end{array}
$$

With the ellipse specified as the source to mirror pole distance defined as entrance arm length $r$ and $r{ }^{\prime}$ defined as the exit arm length between mirror pole and focal point at the experimental position, and the incidence angle $\theta$ of the photons at the mirror pole - see also Fig. 4. In our case the source point of both the horizontal and vertical focusing mirrors is at the nominal source point in the XFEL undulator section of $915.484 \mathrm{~m}$ distance to the vertical focusing mirror pole and of $916.584 \mathrm{~m}$ distance to the horizontal focusing mirror pole.

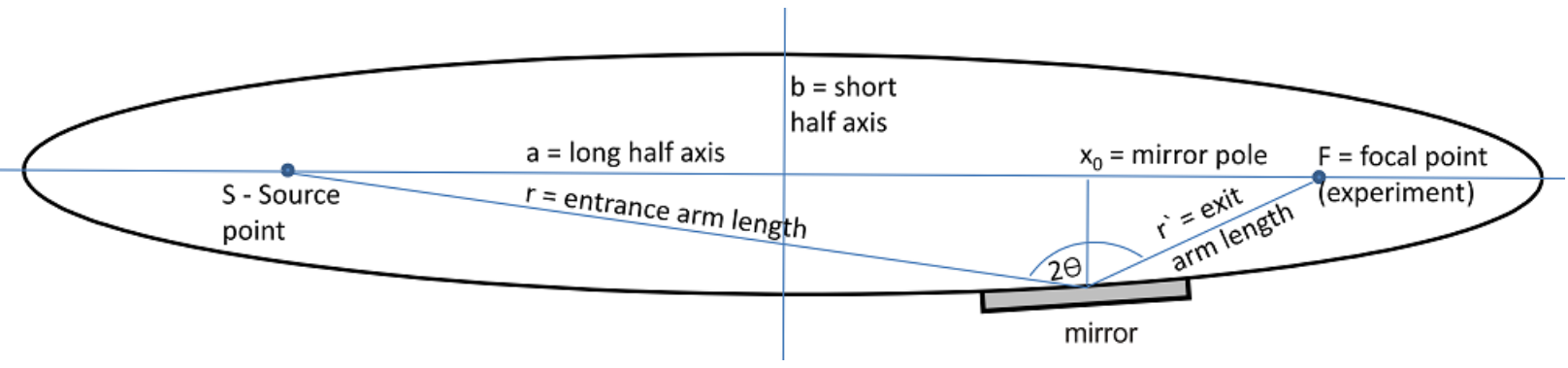

FIG.4. Ideal ellipse for a mirror imaging a source point $\mathrm{S}$ at a distance $\mathrm{r}$ to an image point in a distance $\mathrm{r}^{\prime}$ with the photons reflecting under incidence angle $\theta$ at the mirror pole.

Additional to the linescan measurements we have performed a 2D-Slope mapping to enable a better characterization of the full aperture regarding a possible periodic shape error or local deviations from the ideal shape [45]. Such measurements can be performed in the face-up condition at the BESSY-NOM only. In the tangential scan direction we have chosen an increment of $\Delta \mathrm{x}=0.5 \mathrm{~mm}$ and in the sagittal direction $\Delta \mathrm{y}=1.0 \mathrm{~mm}$ for the two measurement on the vertical focusing mirror and $\Delta y=0.5 \mathrm{~mm}$ for the horizontal focusing mirror.

\section{Measurement results}


Table 1 shows the mirror design parameters as well as the measurement results. While the measured ellipse parameters are in excellent agreement with the specification and show only negligible deviations to the ideal case, the mirrors' topography shows a deviation to the specification in both in terms of the rms slope error as well as the residual figure error. For both mirrors the slope error is of about $100 \mathrm{nrad}$ rms as measured along the mirror central axis. The vertical focusing mirror is characterized by a residual figure error of about $20 \mathrm{~nm} \mathrm{pv} / 6.2 \mathrm{~nm} \mathrm{rms}$ for the state with gauge pieces at the mirror mechanics support position. A deviation of a factor of 10 larger compared to the mirror specification. In the full contact state a significant larger residual of $190.9 \mathrm{~nm} \mathrm{pv} / 61.2 \mathrm{~nm}$ rms is measured (see Fig. 5). Which illustrates a useful pre-shaping of the mirror substrate to compensate the gravitational sag of the substrate to approach the desired mirror shape. Figure 6 shows the results of 2D-slope mapping for both states (full contact support and placed at the gauge pieces) in terms of 2D-slopes in the tangential direction and the corresponding topography in terms of height. Figure 7 is a cut-out of $16 \times 16 \mathrm{~mm}^{2}$ taken from the mirror pool point region at the mirror center. It shows some periodicity in the order of two millimeter spatial frequency. For this see also the PSD-curves at Fig. 10. The horizontal focusing mirror shows a residual figure error of $11.7 \mathrm{~nm} \mathrm{pv} /$ $3.1 \mathrm{~nm}$ rms which is about a factor of 6 higher than specified (see Fig. 8). Fig. 8 shows the profiles of residual slope as achieved for both cases measured: the "upstream to downstream" and "downstream to upstream" case respectively. Both curves are superimposed. The difference between the two curves is of $65 \mathrm{nrad}$ rms. This value can be taken as estimation for the uncertainty achievable for measurements on such very long slightly curved X-ray mirrors. The final result is achieved by averaging of both measurements (black graph). The result of 2D-slope mapping for this mirror is given in Fig.9. 


\begin{tabular}{|c|c|c|c|}
\hline Mirror parameter & Specification & $\begin{array}{l}\text { Measurement by } \\
\text { manufacturer }\end{array}$ & $\begin{array}{c}\text { Result of NOM } \\
\text { measurement }\end{array}$ \\
\hline \multicolumn{4}{|c|}{ Horizontal focusing mirror } \\
\hline Slope error & $\begin{array}{c}\leq 20 \mathrm{nrad} \text { rms } \\
(\leq 100 \mathrm{nrad} \text { rms acceptable })\end{array}$ & $62 \mathrm{nrad} \mathrm{rms}$ & 93nrad rms \\
\hline Residual figure error & $\leq 2.0 \mathrm{~nm} \mathrm{pv}$ & $1.646 \mathrm{~nm} \mathrm{pv}$ & $11.7 \mathrm{~nm} \mathrm{pv} / 3.1 \mathrm{~nm} \mathrm{rms}$ \\
\hline \multicolumn{4}{|l|}{ Parameter of ellipse: } \\
\hline Entrance arm length & $915.484 \mathrm{~m}$ & $915.484 \mathrm{~m}$ & $915.484 \mathrm{~m}$ \\
\hline Exit arm length & $3.30 \mathrm{~m}$ & $3.3 \mathrm{~m}$ & $3.3 \mathrm{~m}$ \\
\hline Grazing angle & $3.5 \mathrm{mrad}$ & $3.5 \mathrm{mrad}$ & $3.457 \mathrm{mrad}$ \\
\hline \multicolumn{4}{|c|}{ Vertical focusing mirror } \\
\hline Slope error & $\begin{array}{c}\leq 20 \mathrm{nrad} \text { rms } \\
(\leq 100 \mathrm{nrad} \text { rms acceptable })\end{array}$ & $68 \mathrm{nrad} \mathrm{rms}$ & 99nrad rms \\
\hline Residual figure error & $\leq 2.0 \mathrm{~nm} \mathrm{pv}$ & $1.821 \mathrm{~nm} \mathrm{pv}$ & $19.2 \mathrm{~nm} \mathrm{pv} / 6.2 \mathrm{~nm} \mathrm{rms}$ \\
\hline \multicolumn{4}{|l|}{ Parameter of ellipse: } \\
\hline Entrance arm length & $916.584 \mathrm{~m}$ & $916.584 \mathrm{~m}$ & $916.584 \mathrm{~m}$ \\
\hline Exit arm length & $2.20 \mathrm{~m}$ & $2.2 \mathrm{~m}$ & $2.2 \mathrm{~m}$ \\
\hline Grazing angle & $3.5 \mathrm{mrad}$ & $3.5 \mathrm{mrad}$ & $3.58 \mathrm{mrad}$ \\
\hline
\end{tabular}

Table 1 Mirror parameter and measurement results as measured by use of the BESSY-NOM in comparison to specification and measurement results of the manufacturer. 

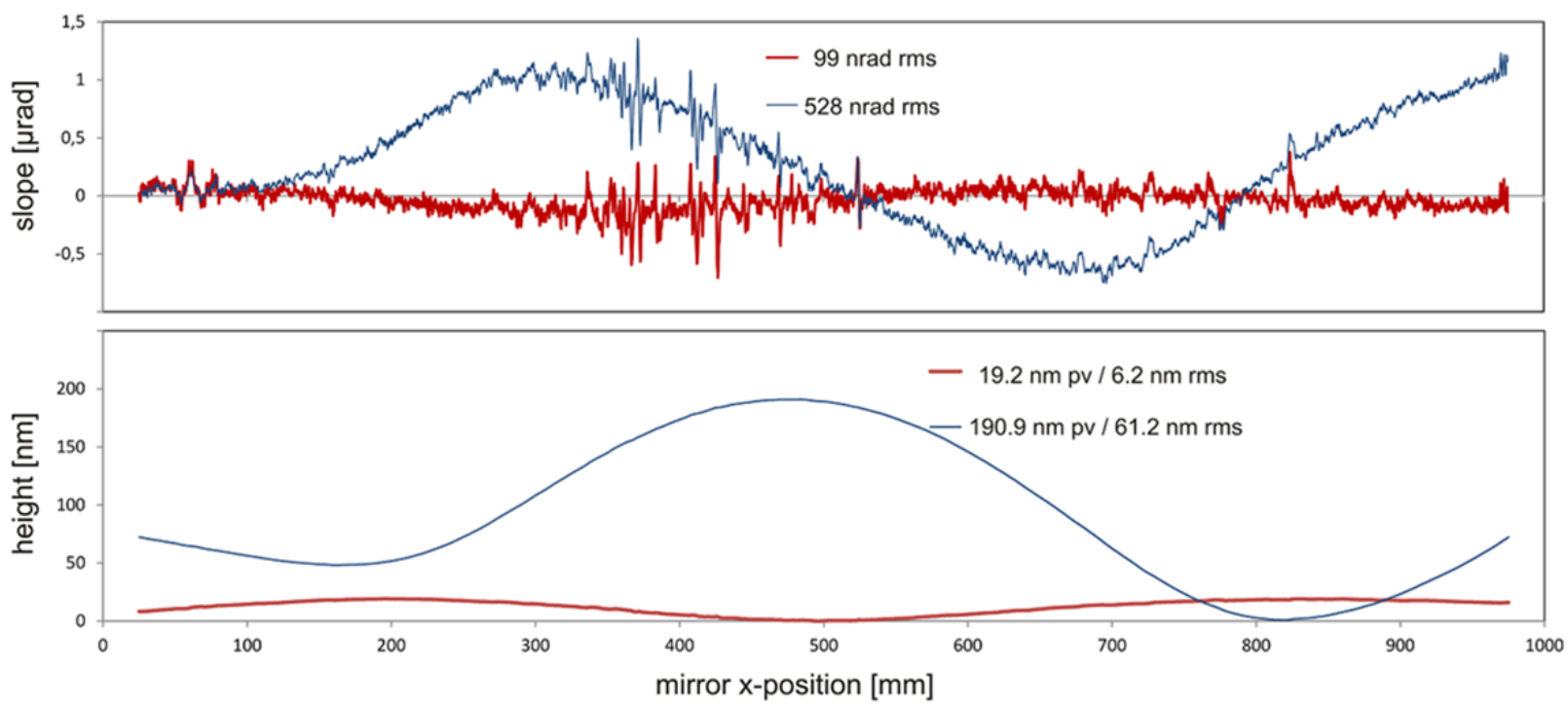

FIG.5. Vertical focusing mirror, top: Profile of residual slope along the mirror central axis in tangential direction for two different state full contact and local support as at the mirror mechanics. Bottom: corresponding profiles of residual height.
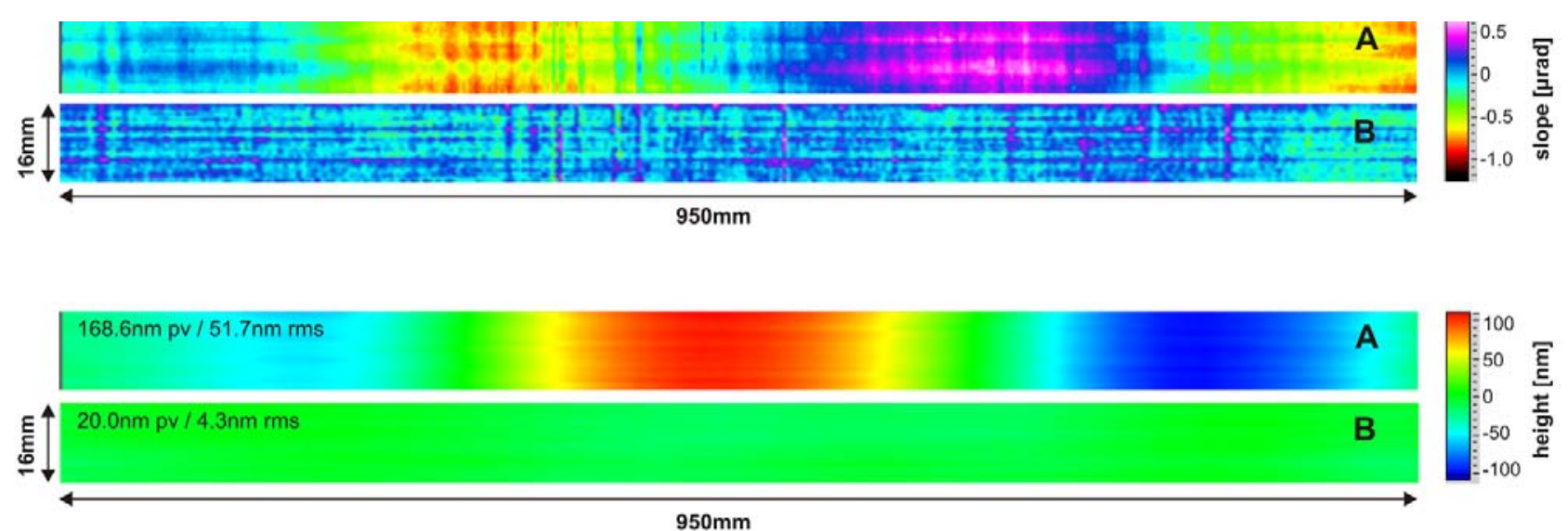

FIG.6. Vertical focusing mirror, top: 2D-slope map in tangential mirror orientation for two different state A full contact and B local support as at the mirror mechanics and bottom corresponding topography in terms of height 

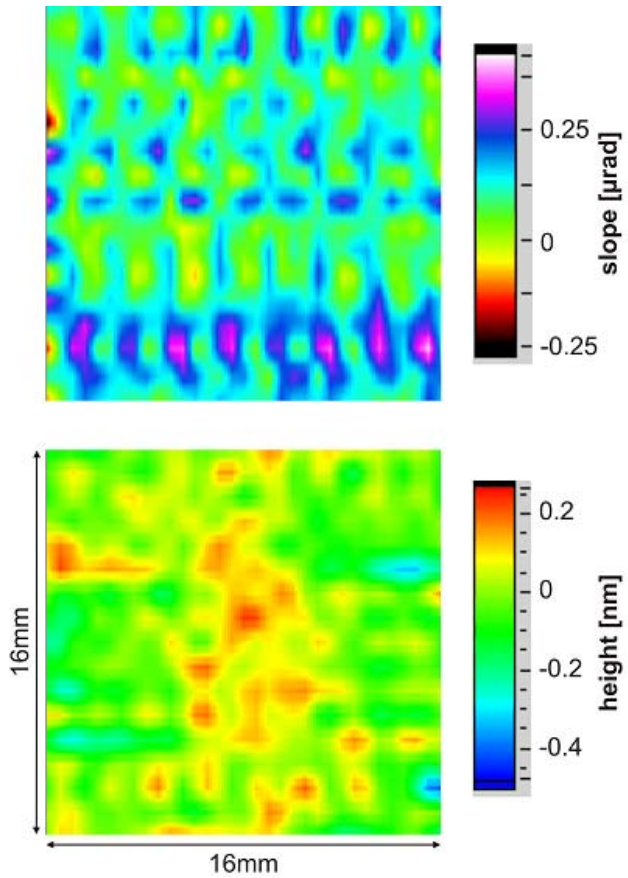

FIG.7. Vertical focusing mirror, $16 \times 16 \mathrm{~mm}^{2}$ cut out at the mirror pool region. The residual figure deviation is of $0.66 \mathrm{~nm} \mathrm{pv} / 0.10 \mathrm{~nm}$ rms for this section.
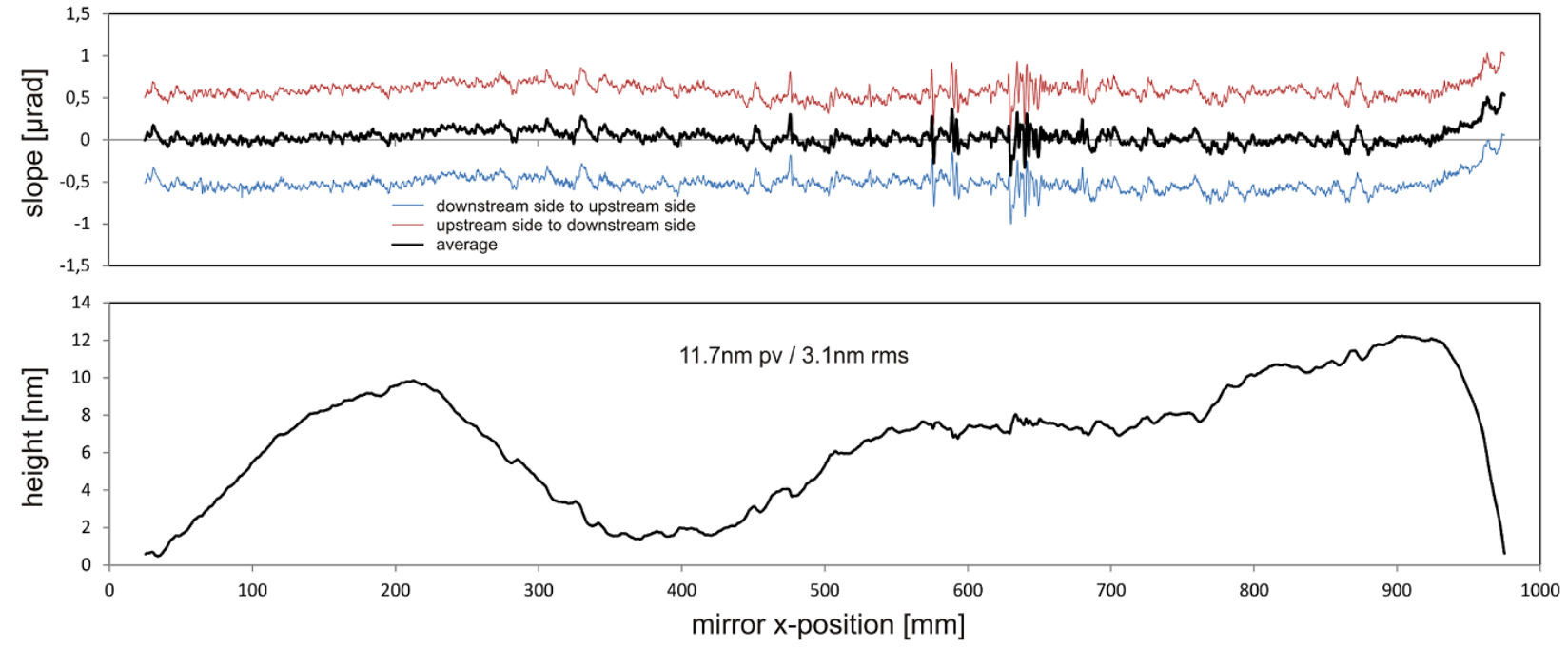

FIG.8. Horizontal focusing mirror, top: Profile of residual slope along the mirror central axis in tangential. Bottom: corresponding profile of residual height. 


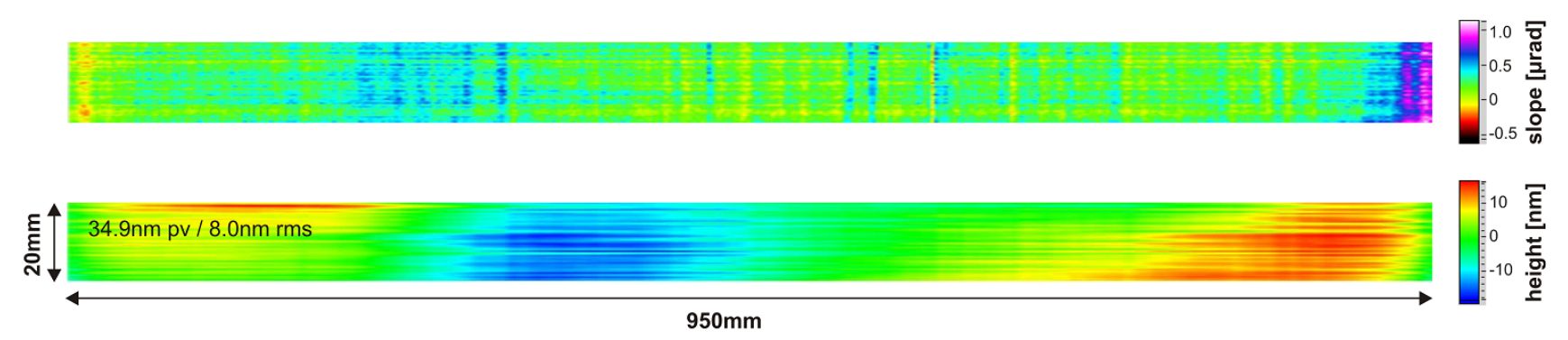

FIG.9. Horizontal focusing mirror, top: 2D-slope map in tangential mirror orientation and bottom corresponding topography in terms of height

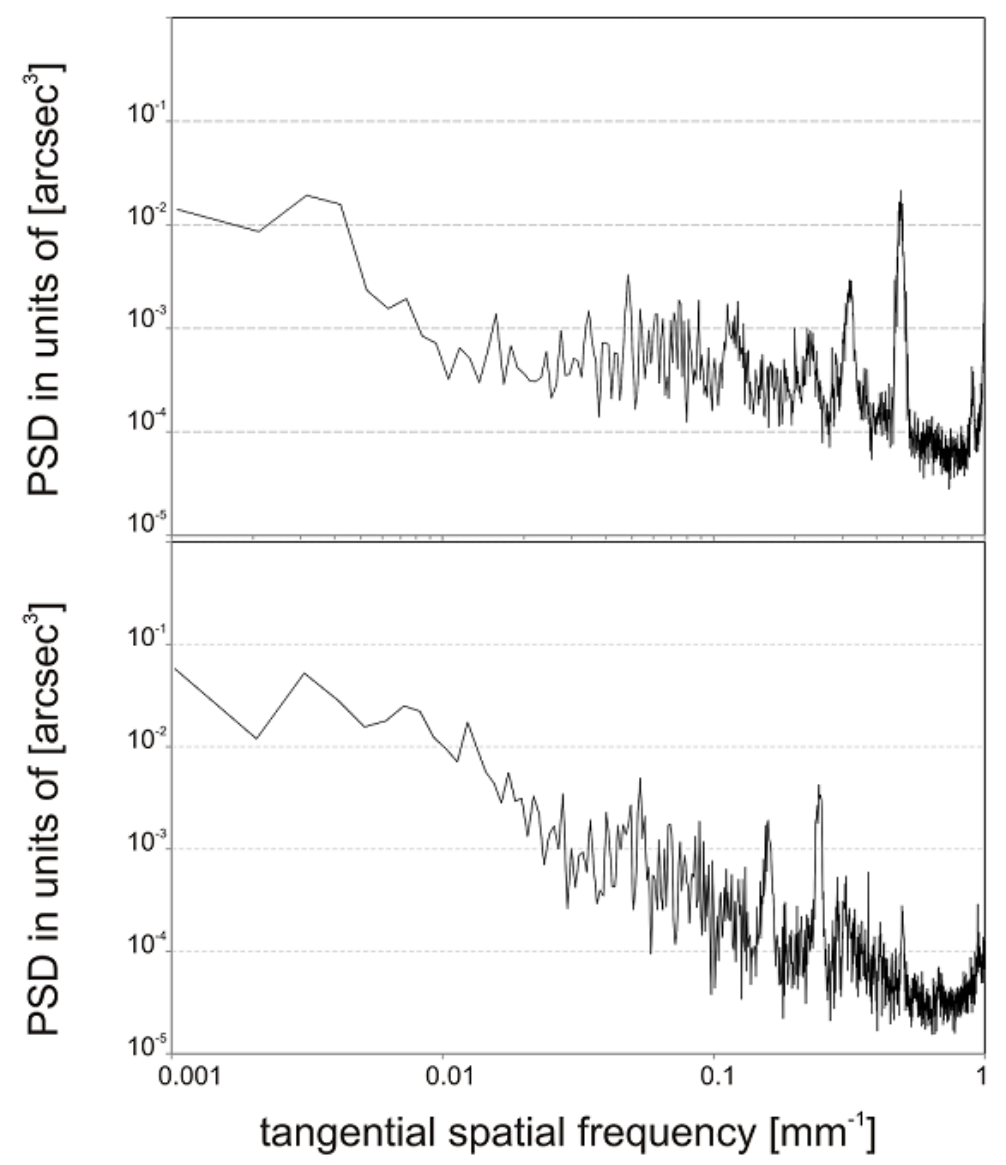

FIG.10. 2D-PSD surface profile as gained from the 2D-slope mapping data (see Fig. 6 and 9), top for the vertical focusing mirror and bottom for the horizontal focusing mirror. 
Fig. 10 depicts the PSD-spectra gained from the 2D-slope mapping of the mirrors in tangential direction. It shows a significant line at a spatial frequency of $2.06 \mathrm{~mm}$ for the vertical focusing mirror and at $4.1 \mathrm{~mm}$ for the horizontal focusing mirror. This periodicity is assumed to have a correlation to the stitching period of the RADSI metrology [46] applied by the manufacturer to measure the mirrors topography and to calculate the removal rate for the EEM finishing technology to polish the mirrors [private communication on this to Kazuto Yamauchi at the SRI Conference in June 2018 in Taipei]. The difference in the periodicity on the two mirrors is due to the different degree of curvature on the mirrors which allows different size of sub-apertures to be measured. Ameliorating such periodic errors, perhaps through additional metrology in the manufacturing process, would lead to significant improvements in the final performance of such mirrors in general.

\section{Conclusion and Outlook}

We have measured and characterized a KB-focusing mirror pair for nano-focusing at the SPB/SFX experimental station at European XFEL. By means of slope measuring deflectometry it is possible to measure such very long mirrors of up to $1 \mathrm{~m}$ in length by line scan as well as 2D-slope mapping in tangential direction. Both mirrors are characterized by a slope error of $100 \mathrm{nrad} \mathrm{rms}$ which corresponds to a residual figure error of $20 \mathrm{~nm} \mathrm{pv}$ for the vertical focusing mirror and of $12 \mathrm{~nm}$ pv for the horizontal focusing mirror respectively. While the figure error in the slope domain is mainly dominated by the higher spatial frequency contribution the height domain shows a low spatial frequency dependency to be dominant. In case of the vertical focusing mirror a pre-shaping of the mirror to compensate the mirror deformation by the gravitational sag is successfully applied. The often mentioned goal of reaching the level of a slope error of $20 \mathrm{nrad}$ rms in the future seems to be presently out of reach, largely due to stitching error contributions in the metrology. However even slope-measuring deflectometry is limited to about $65 \mathrm{nrad} \mathrm{rms}$ as shown in this work. Both mirrors show an excellent agreement with the specification for the parameters of ellipse like entrance arm and exit arm length and grazing angle. It is also noted here that the results discussed in this publication have been achieved under dedicated laboratory condition. The thermal stability at the BESSY-NOM are of $<20 \mathrm{mK} / 24 \mathrm{~h}$, the humidity is of $\pm 5 \% / 24 \mathrm{~h}$ stable and the level of vibration and air convection noise is very low. In addition we have measured the mirrors free of external forces. Thus dedicated care is required for the final mounting and clamping of the mirrors at the instrument to avoid a deformation to the mirrors' aperture. Upcoming work will allow to simulate the expected instrument performance based on this measurement data as realized by previous work [47]. The results of these simulations will be discussed and published elsewhere. 


\section{ACKNOWLEDGMENTS}

The authors are grateful to Akihito Ueda and Hiromi Okada (JTEC-corporation Osaka) for co-working on the mirror polishing and to Kazuto Yamauchi (Osaka University) for very useful discussions during the SRI 2018 in Taipei (Taiwan).

\section{REFERENCES}

${ }^{1}$ R. J. Bean, A. Aquila, L. Samoylova and A. P. Mancuso, "Design of the mirror optical systems for coherent diffractive imaging at the SPB/SFX instrument of the European XFEL", J. Opt. 18 (2016) 074011

${ }^{2}$ R. A. P. Mancuso, A. Aquilla, G. Borchers, K. Giewekemeyer, N. Reimers, “Technical Design Report: Scientific Instrument Single Particles, Clusters, and Biomolecules (SPB)”, XFEL.EU Technical Report 1-232 (2013) [10.3204/XFEL.EU/TR-2013-004]

${ }^{3}$ M. Altarelli, "The European X-ray free-electron laser facility in Hamburg", Nucl. Instrum. Meth. B 269 (2011) 2845-2849

${ }^{4}$ P. Kirkpatrick and A.V. Baez, "Formation of Optical Images by X-Rays", JOSA, 38, 9, (1948)

${ }^{5}$ L. Samoylova et al., "Requirements on hard x-ray grazing incidence optics for European XFEL: analysis and simulation of wavefront transformations" Proc. SPIE 7360, 73600E5 (2009)

${ }^{6}$ Mingwu Wen, Igor V. Kozhevnikov, Frank Siewert, Aleksey V. Buzmakov, Chun Xie, Qiushi Huang, Zhanshan Wang, Liubov Samoylova and Harald Sinn, "Effect of the surface roughness on X-ray absorption by mirrors operating at extremely small grazing angles", Vol. 26, No. 16 | 6 Aug 2018 | OPTICS EXPRESS 21004, doi.org/10.1364/OE.26.021003

${ }^{7}$ K. Yamauchi, H. Mimura, K. Inagaki and Y. Mori, "Figuring with sub-nanometer-level accuracy by numerically controlled elastic emission machining", Rev. Sci. Instrum., 73 (11) (2002)

${ }^{8}$ Michael Störmer, Frank Siewert, Jerome Gaudin, "Development of $x$-ray optics for advanced research light sources", Proc. of SPIE Vol. 8078, Bellingham, WA, doi: 10.1117/12.887530, (2011)

${ }^{9}$ Michael Störmer, Frank Siewert and Harald Sinn, "Preparation and characterization of B4C coatings for advanced research light sources", J. Synchrotron Rad. (2016). 23, http://dx.doi.org/10.1107/S1600577515020901

${ }^{10}$ Michael Störmer, Frank Siewert Christian Horstmann, Jana Buchheim and Grzegorz Gwalt "Coatings for FEL optics: preparation and

characterization of $B_{4} C$ and Pt “, J. Synchrotron Rad. (2018). 25, 116-122, https://doi.org/10.1107/S1600577517016095

${ }^{11}$ A. Aquilla, R. Sobierajski, C. Ozkan, V. Hájková, T. Burian, J. Chalupský, L. Juha, M. Störmer, S. Bajt, M. T. Klepka, P. Dłużewski, K. Morawiec, H. Ohashi, T. Koyama, K. Tono, Y. Inubushi, M. Yabashi, H. Sinn, T. Tschentscher, A. P. Mancuso, and J. Gaudin, "Fluence thresholds for grazing incidence hard x-ray mirrors", Appl. Phys. Lett. 106, 241905 (2015); https://doi.org/10.1063/1.4922380

${ }^{12}$ F. Siewert, J. Buchheim, T. Zeschke, M. Störmer, G. Falkenberg and R. Sankari, On the characterization of ultra-precise X-ray optical components: advances and challenges in ex situ metrology, J. Synchrotron Rad. 21, 968-975 (2014), doi:10.1107/S1600577514016221

${ }^{13}$ G. Ehret, M. Schulz, M. Stavridis and C. Elster, Deflectometric systems for absolute flatness measurements at PTB, Meas. Sci. Technol. 23 (2012) 094007 (8pp), doi:10.1088/0957-0233/23/9/094007

${ }^{14}$ P. Takacs, S. N. Qian and J. Colbert, Design of a long trace surface profiler, Proc. of SPIE Vol. 749, Bellingham, WA, pp. 59-64 (1987)

${ }^{15}$ Valeriy V.Yashchuk, PeterZ.Takacs, WayneR.McKinney, LahsenAssoufid, Frank Siewert, ThomasZeschke, Development of a new generation of optical slope measuring profiler, Nucl. Instrum. Meth. A 649 (2011) 153-155

${ }^{16}$ Frank Siewert, Tino Noll, Thomas Schlegel, Thomas Zeschke, and Heiner Lammert, The Nanometer Optical Component Measuring machine: a new Subnm Topography Measuring Device for X-ray Optics at BESSY, AIP Conference Proceedings 705, American Institute of Physics, Mellville, NY, 2004, pp. $847-850$

${ }^{17}$ F. Siewert, Slope Error and Surface Roughness, in: Modern Developments in X-ray and Neutron Optics, Springer 2008

${ }^{18}$ Henry N. Chapman et al, "Femtosecond X-ray protein nanocrystallography", Nature Vol. 470/73 (2011), doi:10.1038/nature09750

${ }^{19}$ M. Levantino, G. Schiro, HT. Lemke, G. Cottone, JM. Glownia, D. Zhu, M. Chollet, H. Ihee, A. Cupane and M. Cammarata, "Ultrafast myoglobin structural dynamics observed with an X-ray free-electron laser", Nature Communications, (2015), | 6:6772 | DOI: 10.1038/ncomms7772 |

${ }^{20}$ A. Aquila, A. Barty, C. Bostedt, S. Boutet, G. Carini, D. dePonte, P. Drell, S. Doniach, K. H. Downing, T. Earnest, H. Elmlund, V. Elser, M. Gühr, J. Hajdu, J. Hastings, S. P. Hau-Riege, Z. Huang, E. E. Lattman, F. R. N. C. Maia, S. Marchesini,6 A. Ourmazd, C. Pellegrini, R. Santra, I. Schlichting, C. Schroer, J. C. H. Spence, I. A. Vartanyants, S. Wakatsuki, W. I. Weis, and G. J. Williams,"The linac coherent light source single particle imaging road map", Struct Dyn. 2015 Jul; 2(4): 041701, doi: 10.1063/1.4918726

${ }^{21}$ A.P. Mancuso, “Conceptual Design Report: Scientific Instrument Single Particles, Clusters, and Biomolecules (SPB)”, Technical Report (2011) [10.3204/XFEL.EU/TR-2011-007], doi:10.3204/XFEL.EU/TR-2011-007

${ }^{22}$ M.F. Hantke, et al, "High-throughput imaging of heterogeneous cell organelles with an X-ray laser" Nature Photonics (2014), doi: 10.1038/NPHOTON.2014.270

${ }^{23}$ X. Shi, R. Dinapoli, B. Henrich, A. Mozzanica, B. Schmitt, R. Mazzocco, H.Krüger, U. Trunk, H. Graafsma, "Challenges in chip design for the AGIPD detector", Nucl. Instrum. Meth. A 624 (2010) 387-391, doi: 10.1016/j.nima.2010.05.038

${ }^{24}$ M. Altarelli, "From 3rdto 4thGeneration Light Sources: FreeElectron Lasers in the Xray Rangel”, Crystallography Reports, 2010, Vol. 55, No. 7, pp. $1145-1151$

${ }^{25}$ M.O. Wiedorn et al, "Rapid sample delivery for megahertz serial crystallography at X-ray FELs“,IUCrJ (2018).5, 574-584 doi.org/10.1107/S2052252518008369

${ }^{26}$ M.L. Grünbein, J. Bielecki, A. Gorel, M. Stricker, R. Bean, M. Cammarata, K. Dörner, L. Fröhlich, E. Hartmann, S. Hauf, M. Hilpert, Y. Kim, M. Kloos, R. Letrun, M. Messerschmidt, G. Mills, G. Nass Kovacs, M. Ramilli, C.M. Roome, T. Sato, M. Scholz, M. Sliwa, J. Sztuk-Dambietz, M. Weik, B. Weinhausen, N. Al-Qudami, D. Boukhelef, S. Brockhauser, W. Ehsan, M. Emons, S. Esenov, H. Fangohr, A. Kaukher, T. Kluyver, M. Lederer, L. Maia, M. Manetti, T. Michelat, A. Münnich, F. Pallas, G. Palmer, G. Previtali, N. Raab, A. Silenzi, J. Szuba, S. Venkatesan, K. Wrona, J. Zhu, R.B. Doak, R.L. Shoeman, L.Foucar, J.-P. Colletier, A.P. Mancuso, T.R.M. Barends, C.A. Stan and I. Schlichting, "Megahertz data collection from protein microcrystals at an X-ray free-electron laser", NATURE COMMUNICATIONS (2018) 9:3487 | DOI: 10.1038/s41467-018-05953-4

${ }^{27}$ F.Siewert, J. Buchheim, T. Zeschke, Characterization and calibration of 2nd generation slope measuring profiler, Nucl. Instrum. Meth. A 616 pp.118-127 (2010), doi:10.1016/j.nima.2009.12.033

${ }^{28}$ E. Debler, K. Zander, Ebenheitsmessung an optischen Planflächen mit Autokollimationsfernrohr und Pentagonprisma, PTB Mitteilungen Forschen+ Prüfen, Amts und Mitteilungsblatt der Physikalisch Technischen Bundesanstalt, Braunschweig und Berlin, 1979, pp. 339-349.

${ }^{29}$ S. Qian, W. Jark P.Z. Takacs, "The pentaprism LTP - a long trace profiler with stationary optical head and moving penta prism", Review of Scientific Instruments, Volume: 66, 3 pp: 2562-2569 (1995) 
${ }^{30}$ S.G. Alcock, K.J.S. Sawhney, S. Scott, U. Pedersen, R. Walton, F. Siewert, T. Zeschke, T. Noll and H. Lammert, The Diamond-NOM: A non-contact profiler capable of characterizing optical figure error with sub-nm repeatability, Nucl. Instrum. Meth. A 616 (2010) 224-228

${ }^{31}$ V.V. Yashchuk, S. Barber, E.E. Domning, J.L. Kirschman, G.Y. Morrison, B.V. Smith, F. Siewert, T. Zeschke, R. Geckler, A. Just, Sub-microradian surface slope metrology with the ALS Developmental Long Trace Profiler, Nucl. Instrum. Meth. A 616 (2010) 212-223

${ }^{32}$ R.D. Geckeler, I. Weingärtner, Sub-nm topography measurement by deflectometry: Flatness standard and wafer nanotopography, Proc. of SPIE, Volume 4779, pp. 1-12 (2002)

${ }^{33}$ Moeller-Wedel Optical GmbH, Rosengarten 10, D-22880 Wedel, Germany, http://www.moeller-wedel-optical.com

${ }^{34}$ Gerald Fütterer, Simulation of detectors response of an autocollimator, in: Proceedings of the SPIE, Bellingham, WA, vol. 6617,2007

${ }^{35}$ F.Siewert, J. Buchheim, T. Zeschke, G. Brenner, S. Kapitzki, K. Tiedtke, Sub-nm accuracy metrology for ultra-precise reflective X-ray optics, Nucl. Instrum. Meth. A 635 pp.52-57 (2011)

${ }^{36}$ S. K. Barber, G.Y. Morrison,V.V.Yashchuk, M.V. Gubarev, R. D. Geckeler, J. Buchheim, F. Siewert, and T. Zeschke, "Developmental long trace profiler using optimally aligned mirror based pentaprism" Opt. Eng. 50(5), 053601 (2011).

${ }^{37}$ S. K. Barber, R. D. Geckeler, V.V.Yashchuk, M.V. Gubarev, J. Buchheim, F. Siewert, and T. Zeschke, "Optimal alignment of mirror-based pentaprisms for scanning deflectometric devices" Opt. Eng. 50(7), 073602 (2011)

${ }^{38}$ V. Yashchuk, W. McKinney, T. Warwick, T. Noll, F. Siewert, T. Zeschke, R. Geckeler, Proposal for a Universal Test Mirror for Characterization of Slope Measuring Instruments, Advances in Metrology for X-Ray and EUV Optics II, Proc. of SPIE, Vol. 6704, Bellingham, WA, 2007

${ }^{39}$ Valeriy V. Yashchuk, Optimal measurement strategies for effective suppression of drift errors, Rev. Sci. Instrum., 80, 115101 (2009)

${ }^{40} \mathrm{~F}$. Siewert, J. Buchheim, T. Höft, T. Zeschke, A. Schindler, T. Arnold, Investigations on the spatial resolution of autocollimator-based slope measuring profilers, Nucl. Instrum. Methods Phys. Res. A 710 42-47 (2013)

${ }^{41}$ F. Siewert, T. Zeschke, T. Arnold, H. Paetzelt, and V. V. Yashchuk, Linear chirped slope profile for spatial calibration in slope measuring deflectometry, Review of Scientific Instruments 87, 051907 (2016); doi: 10.1063/1.4950737

${ }^{42}$ S.C. Irick, Rev. Sci. Instrum. 631 (1992)

${ }^{43}$ F. Siewert, J. Buchheim, S. Boutet, G. Williams, P. A. Montanez, J. Krzywinski, R. Signorato, Ultra-precise characterization of LCLS hard X-ray focussing mirrors by high resolution slope measuring deflectometry, Optics Express, Vol. 20, No. 4 (2012) 4525-4536

${ }^{44}$ J.P. Sutter, M. Amboage, S. Hayama, S. Diaz-Moreno, "Geometrical and wave-optical effects on the performance of a bent-crystal dispersive X-ray spectrometer", Nucl. Instr. and Meth. A 621 (2010) pp 627-636

${ }^{45} \mathrm{~F}$. Siewert et al., "Advanced metrology: an essential support for the surface finishing of high performance x-ray optics," Proc. SPIE 5921, 592101 (2005).

${ }^{46}$ Kazuto Yamauchi, Kazuya Yamamura, Hidekazu Mimura, Yasuhisa Sano, Akira Saito, Kazumasa Ueno, Katsuyoshi Endo, Alexei Souvorov and Makina Yabashi, Kenji Tamasaku and Tetsuya Ishikawa, Yuzo Mori, Microstitching interferometry for x-ray reflective optics, Review of Scientific Instruments 74 (5), (2003), DOI: 10.1063/1.1569405

${ }^{47} \mathrm{~S}$. Roling et al., "Time-dependent wave front propagation simulation of a hardx-ray split-and-delay unit: towards a measurement of the temporal coherence properties of x-ray free electron lasers” Phys. Rev. ST Accel. Beams 17, 110705 (2014) 


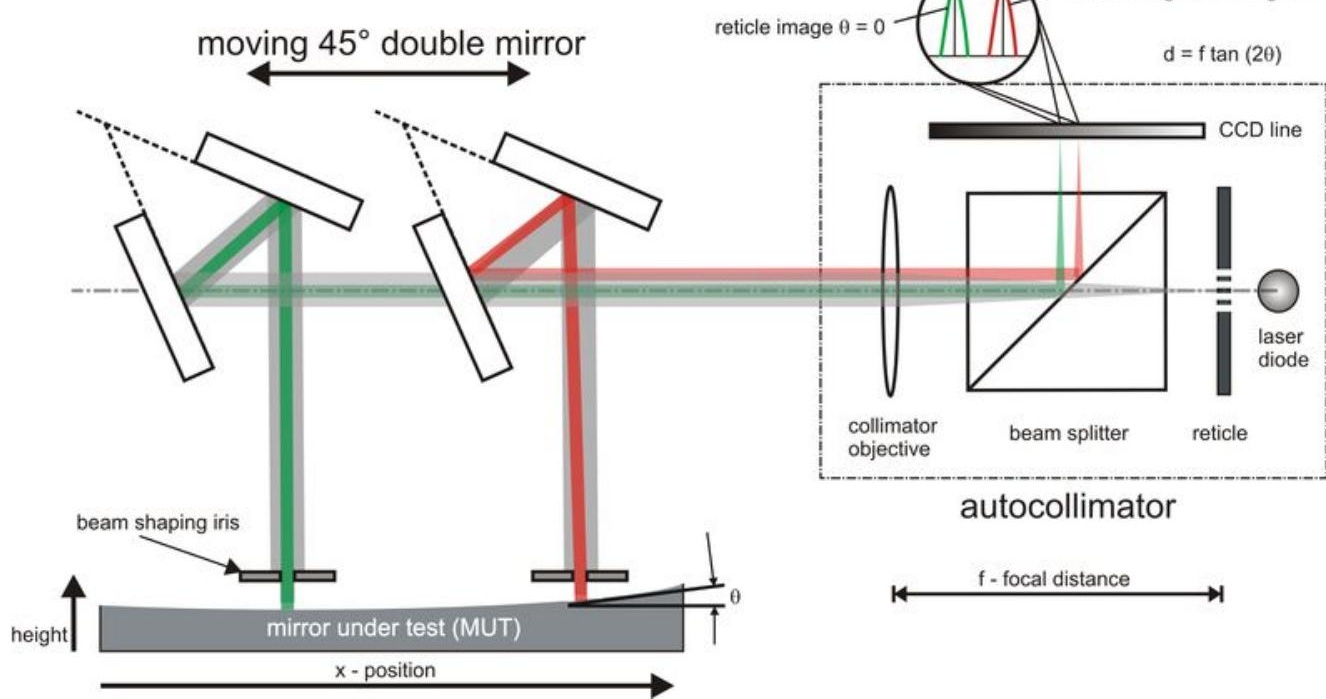




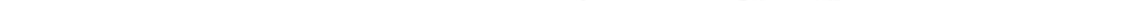


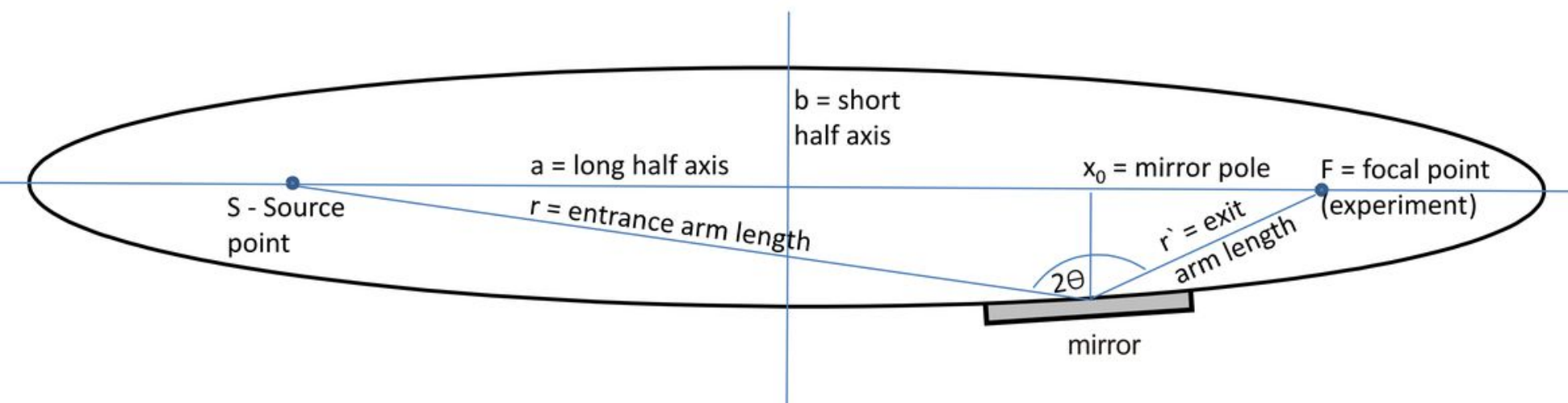



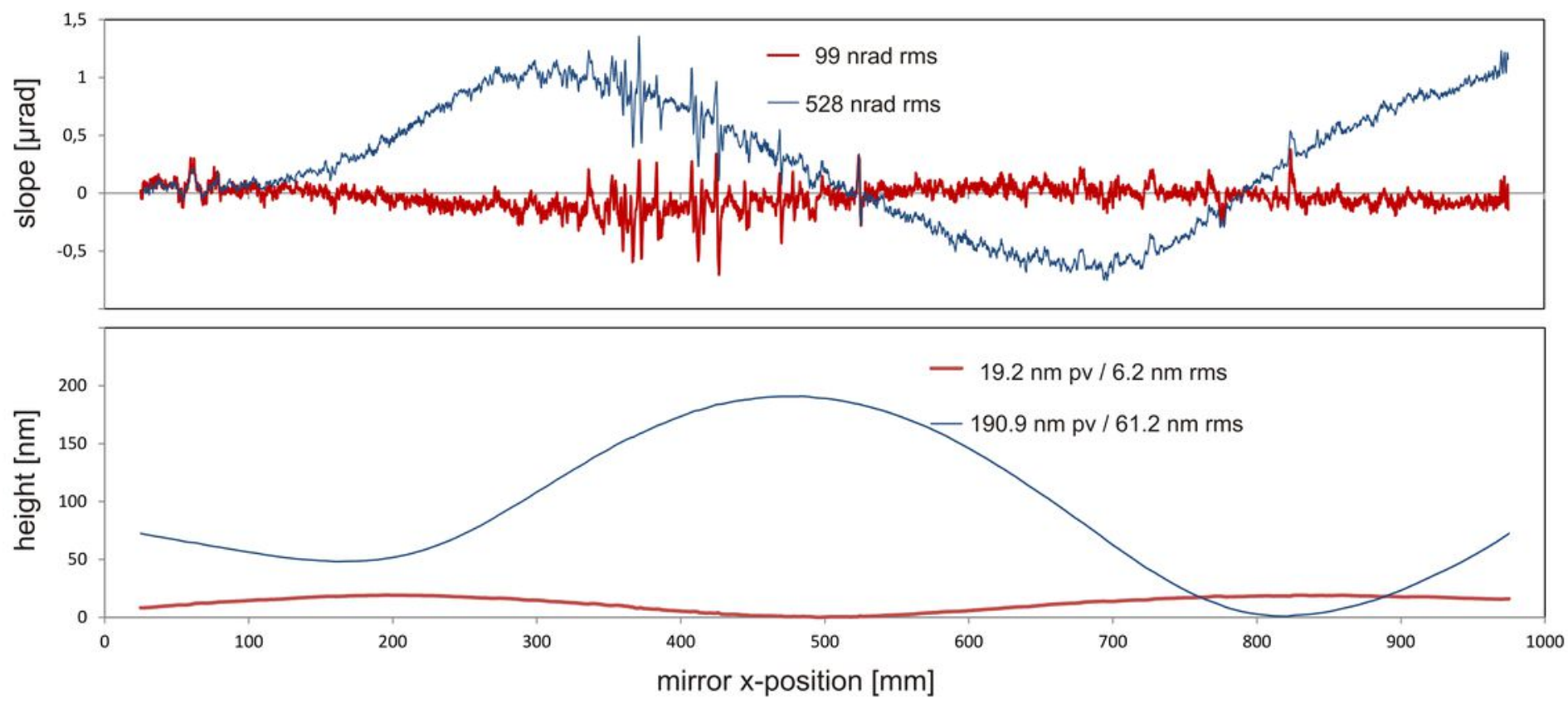


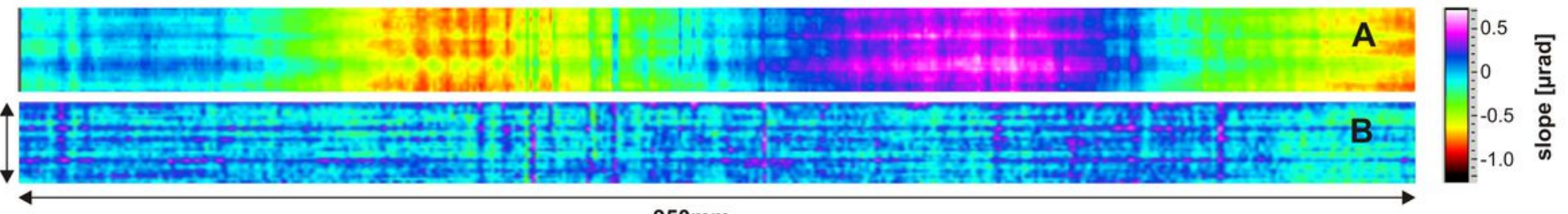

$950 \mathrm{~mm}$

$168.6 \mathrm{~nm}$ pv / $51.7 \mathrm{~nm} \mathrm{rms}$

E્
A

B 

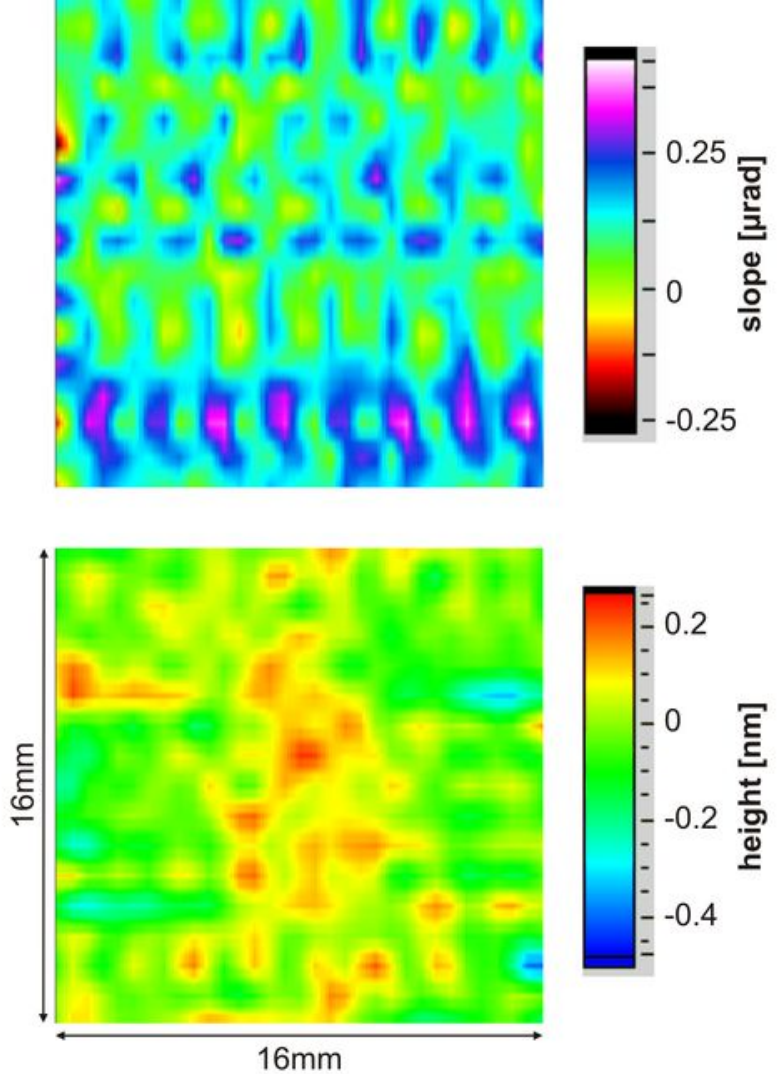


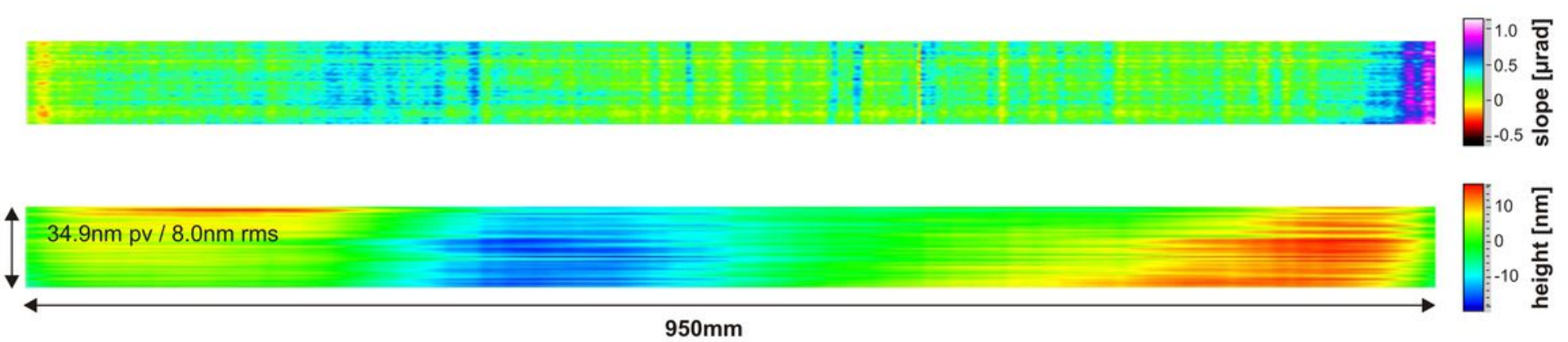




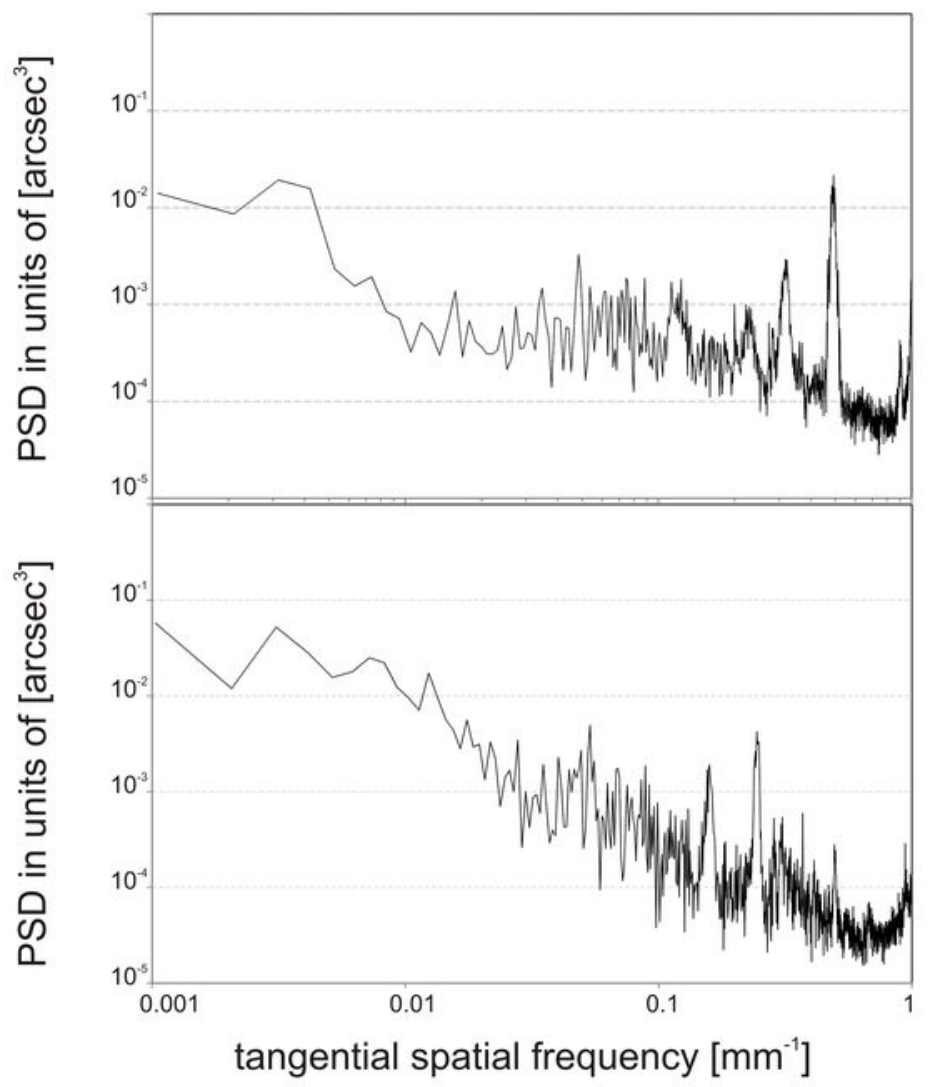

